\title{
Utilization of green fluorescent protein as a marker for studying the expression and turnover of the monocarboxylate permease Jen1p of Saccharomyces cerevisiae
}

\author{
Sandra PAIVA*, Arthur L. KRUCKEBERG $†$ and Margarida CASAL*1 \\ ${ }^{*}$ Centro de Ciências do Ambiente, Departamento de Biologia, Universidade do Minho, 4710-057 Braga Codex, Portugal, and †Swammerdam Institute of Life Science, \\ The University of Amsterdam, Plantage Muidergracht, 12 NL-1018 TV Amsterdam, The Netherlands
}

Green fluorescent protein (GFP) from Aequorea victoria was used as an in vivo reporter protein when fused to the C-terminus of the Jen1 lactate permease of Saccharomyces cerevisiae. The Jen 1 protein tagged with GFP is a functional lactate transporter with a cellular abundance of 1670 molecules/cell, and a catalyticcentre activity of $123 \mathrm{~s}^{1}$. It is expressed and tagged to the plasma membrane under induction conditions. The factors involved in proper localization and turnover of Jen $1 \mathrm{p}$ were revealed by expression of the Jen $1 \mathrm{p}-$ GFP fusion protein in a set of strains bearing mutations in specific steps of the secretory and endocytic pathways. The chimaeric protein Jen $1 \mathrm{p}-\mathrm{GFP}$ is targeted to the plasma membrane via a Sec6-dependent process; upon treatment with glucose, it is endocytosed via END3 and targeted for degradation in the vacuole. Experiments performed in a $\Delta d o a 4$ mutant strain showed that ubiquitination is associated with the turnover of the permease.

Key words: yeast, transport, secretion, ubiquitination, endocytosis.

\section{INTRODUCTION}

It has been shown that the product of the gene JEN1 is required for the uptake of lactate and other monocarboxylates in the yeast Saccharomyces cerevisiae [1]. To date, this is the first and only gene known to be involved in monocarboxylate transport in fungi. An exhaustive study carried out recently revealed that the $S$. cerevisiae homologues of mammalian monocarboxylate permeases do not transport monocarboxylic acids across the plasma membrane [2], in contrast with what had been suggested from computer analyses $[3,4]$. Jen $1 p$ is therefore the only model of a monocarboxylate transporter in yeast, and its study can provide important insights into the function of the mammalian monocarboxylate transporters ('MCTs'; for a review, see [5]), and of permeases in general. In the case of plasma membrane proteins, an effort is being orchestrated by several groups towards the understanding of their trafficking, localization and degradation. Previous studies performed in our laboratory have shown that $J E N 1$ expression is mediated under glucose catabolite repression at distinct levels: transcription, mRNA turnover and carrier inactivation [6]. Jen $1 \mathrm{p}$ is rapidly inactivated on addition of glucose to induced cells, undergoing an irreversible catabolicinactivation process [6]. In the present study, green fluorescent protein (GFP) (for a recent review, see [7]) from Aequorea victoria was used as in vivo reporter protein, fused to the Cterminus of the Jen 1 monocarboxylate permease of $S$. cerevisiae. Our studies focused on the expression, trafficking and turnover of Jen $1 \mathrm{p}$.

\section{MATERIALS AND METHODS}

Strains, plasmids and growth conditions

The $S$. cerevisiae strains used in this study are listed in Table 1 . Plasmid pFA6a-GFPS65T-KanMX6, kindly provided by A. Wach (Institut für Angewandte Mikrobiologie, Biozentrum,
Universität Basel, Basel, Switzerland), was used for the construction of the JEN1-GFP chimaera. The plasmid YEp96, which contains a synthetic yeast Ubiquitin $(U b)$ gene under the control of the copper-inducible CUPI promoter, was used [8] for the overexpression of $\mathrm{Ub}$. The cultures were maintained on slants of $1 \%(\mathrm{w} / \mathrm{v})$ yeast extract, $1 \%(\mathrm{w} / \mathrm{v})$ peptone, $2 \%(\mathrm{w} / \mathrm{v})$ glucose and $2 \%(\mathrm{w} / \mathrm{v})$ agar. To promote growth, either a complex medium containing $1 \%(\mathrm{w} / \mathrm{v})$ yeast extract and $1 \%$ $(\mathrm{w} / \mathrm{v})$ peptone (YP medium) or a synthetic minimal medium comprising $0.67 \%$ (w/v) Difco yeast nitrogen base, supplemented with adequate quantities of auxotrophic requirements (YNB medium), were used. Carbon sources were either glucose $(2 \%$, $\mathrm{w} / \mathrm{v})$ or $\mathrm{D}, \mathrm{L}-\mathrm{lactic}$ acid $(0.5 \%, \mathrm{v} / \mathrm{v}$; at $\mathrm{pH} 5.0)$. Strains with temperature-sensitive alleles were grown at $24^{\circ} \mathrm{C}$ (the permissive temperature) or $37^{\circ} \mathrm{C}$ (the restrictive temperature); other strains were grown at $30^{\circ} \mathrm{C}$. Cultures were always harvested

Table 1 Yeast strains used in this paper

\begin{tabular}{|c|c|c|}
\hline Strain & Genotype & Source or reference \\
\hline CEN.PK113-5D & MATa ura3-52 & [8] \\
\hline CEN.PK 113-13D- $\Delta$ jen1 & MATa ura3-52 $\Delta$ jen1 & [2] \\
\hline BLC 491-U2 & MATa ura3-52 JEN1::GFP Kan' ${ }^{\prime}$ & The present work \\
\hline NY17 & MATa sec6-4 ura3-52 & [9] \\
\hline RH1623 & MATa his4 leu2 ura3 bar1-1 end3-1 & [10] \\
\hline BLC 492 & MATa ura3-52 sec6-4 JEN1:: GFP Kan' & The present work \\
\hline BLC 493 & $\begin{array}{l}\text { MATa his4 ura3 bar1-1 end3-1 } \\
\text { JEN1:GFP Kan' }\end{array}$ & The present work \\
\hline MHY501 & $\begin{array}{l}\text { MATa his3- } \Delta 200 \text { leu2-3,112 ura3-52 } \\
\text { lys2-801 trp1 }\end{array}$ & {$[11]$} \\
\hline MHY623 & $\begin{array}{l}\text { MATa his3- } \Delta 200 \text { ura3-52 lys2-801 trp1 } \\
\text { doa4: :LEU2 }\end{array}$ & [11] \\
\hline
\end{tabular}

Abbreviations used: CMAC-Arg, 7-amino-4-chloromethylcoumaryl-L-arginine amide dihydrochloride; GFP, green fluorescent protein; ORF, open reading frame; Ub, ubiquitin.

1 To whom correspondence should be addressed (e-mail mcasal@bio.uminho.pt). 
Table 2 Oligonucleotides used to construct and check the JEN1-GFP fusion

The 21 underlined nt of primer $S 1$ anneal to the $5^{\prime}$-end of the GFP ORF in plasmid pFA6aGFPS65T-KanMX6A. The underlined $19 \mathrm{nt}$ of primer S2 anneal to the $3^{\prime}$-end of the $A D H 1$ terminator in plasmid pFA6a-GFPS65T-KanMX6A.

\begin{tabular}{|c|c|}
\hline Oligonucleotide & Sequence \\
\hline S1 & $\begin{array}{l}\text { 5'-GATTCGAACGTCTCAAAGACATATGAGGAGCATATTGAGAC } \\
\text { CGTTAGTAAAGGAGAAGAACTTTTC-3' }\end{array}$ \\
\hline S2 & $\begin{array}{l}\text { 5'-GTTACATAGAGAAGCGAACACGCCCTAGAGAGCAATGAAAA } \\
\text { GTGAGGATGGCGGCGTTAGTATC-3' }\end{array}$ \\
\hline K2 & 5'-CGATAGATTGTCGCACCTG-3' \\
\hline K3 & 5'-CCATCCTATGGAACTGCCTC-3' \\
\hline $\mathrm{A} 1$ & 5'-GGCCTATCCAAGGATGCTGTC-3' \\
\hline A2 & 5'-GGCCCATTCAGTGCAAGAACC-3' \\
\hline
\end{tabular}

during the exponential phase of growth. Glucose-containing media, i.e. YP (YPD) or YNB (YNBD) were used for the growth of yeast cells under conditions of repression. Conditions for the induction of yeast were obtained by incubating cells previously grown under repression conditions in YNB medium with $\mathrm{D}, \mathrm{L}-$ lactic acid for $4 \mathrm{~h}$. Media contained $0.1 \mathrm{mM} \mathrm{CuSO}_{4}$ for experiments involving overexpression of Ub. Cell growth was monitored by measuring the attenuance $(D)$ of appropriately diluted cell suspensions at $600 \mathrm{~nm}$.

\section{Genetic methods}

Crosses, isolations of diploids, sporulation and tetrad analysis were achieved using standard methods [13].

\section{JEN1-GFP chimaeric DNA fragment and transformation}

A genetic chimaera formed between JEN1 and GFP was made to yield a fusion gene at the chromosomal JEN1 locus. The flanking homology PCR cassette technique $[14,15]$ was used. Primer S1 (Table 2) has 46 bp of DNA that are homologous with the $3^{\prime}$-end of the JEN1 open reading frame (ORF), not including the stop codon, followed by $21 \mathrm{bp}$ of sequence derived from the $5^{\prime}$-end of the GFP reporter gene in the plasmid pFA6a-GFPS65TKanMX6. Primer S2 (Table 2) has 45 bp DNA homologous with first 45 nucleotides downstream of the JEN1 ORF, followed by $19 \mathrm{bp}$ of sequence derived from the $3^{\prime}$-end of the $A D H 1$ terminator in the plasmid pFA6a-GFPS65T-KanMX6A. The resulting PCR product of $2.4 \mathrm{~kb}$ was purified, and used to transform cells of the strain CEN.PK 113-5D by the improved lithiumacetate method [16]. Transformed cells were grown at $30^{\circ} \mathrm{C}$ in YPD media for $4 \mathrm{~h}$ and then spread on to YPD plates containing 200 mg/1 Geneticin (G418 from Life Technologies; Gaithersburg, MD, U.S.A.). To purify transformants from the background, each large colony was re-streaked on to fresh YPD/Geneticin plates. Only those clones that grew after both means of selection were analysed further as potentially the sought-after transformants by analytical PCR, as described by Kruckeberg et al. [17]. Molecular biology techniques were performed using standard procedures published previously [18].

\section{Transport assays}

Cells incubated under de-repressed conditions were harvested by centrifugation, and washed twice in ice-cold deionized water to a final concentration of about $25-40 \mathrm{mg}$ dry weight $/ \mathrm{ml}$. Conical centrifuge tubes containing $30 \mu \mathrm{l}$ of $0.1 \mathrm{M} \mathrm{KH}_{2} \mathrm{PO}_{4}$ buffer at $\mathrm{pH}$ 5.0 and $10 \mu \mathrm{l}$ of the yeast suspension were incubated for $2 \mathrm{~min}$ at $25^{\circ} \mathrm{C}$. The reaction was started by the addition of $10 \mu \mathrm{l}$ of an aqueous solution of 4000 d.p.m./nmol of radiolabelled L-[1${ }^{14}$ C]lactic acid (sodium salt; Amersham Biosciences, Piscataway, NJ, U.S.A.) at $\mathrm{pH}$ 5.0. The reaction was stopped by dilution with $5 \mathrm{ml}$ of ice-cold water. The reaction mixtures were filtered immediately through GF/C membranes (Whatman Biosystems Ltd., Maidstone, Kent, U.K.) and the filters were washed with $10 \mathrm{ml}$ of ice-cold water and transferred to scintillation fluid (Opti-Phase HiSafe II; LKB, Gaithersburg, MD, U.S.A.). Radioactivity was measured in a Packard Tri-Carb 2200 CA liquid scintillation spectrophotometer equipped with a d.p.m. correction facility. For non-specific adsorption of ${ }^{14} \mathrm{C}$, labelled lactic acid was added at time zero after the cold water. To determine the best fitting transport kinetics to the experimental initialuptake rate values, and to estimate the kinetic parameters, a computer-assisted non-linear regression analysis (GraphPAD software; San Diego, CA, U.S.A.) was used. All the experiments were repeated at least three times, and the data reported represent the average values.

\section{Microscopy}

Living cells were examined with a Leitz Aristoplan epifluorescence microscope with filter cube 1001 HQ-FITC (Chroma Technology, Brattleboro, VT, U.S.A.) for GFP excitation, and filter A for 7-amino-4-chloromethylcoumaryl-L-arginine amide dihydrochloride (CMAC-Arg; Molecular Probes, Eugene, OR, U.S.A.). For staining of vacuolar lumen, cells were incubated with CMAC-Arg according to the manufacturer's instructions. For the capture of images, an Apogee charge-coupled-device camera was used, and the micrographs were processed for display using Image Pro Plus software.

\section{Cell protein measurement}

Total cell protein was measured by the Lowry assay, after the digestion of cells overnight in $1 \mathrm{M} \mathrm{NaOH}$.

\section{Fluorescence spectroscopy}

A Hitachi RF-5001PC fluorimeter was used to scan the excitation and emission spectra of whole-cell suspensions. Spectra of cells expressing JEN1-GFP were normalized for cell density, and were corrected for background by subtraction of spectra that were recorded for cells expressing JENI cultured under identical conditions. Emission spectra were collected between 500 and $550 \mathrm{~nm}$, with excitation set at $489 \mathrm{~nm}$, and excitation and emission slit widths of $3 \mathrm{~nm}$.

\section{Catalytic-centre activity and cellular abundance estimation}

The catalytic-centre activity and cellular abundance of Jen1-GFP protein were estimated by the method of Kruckeberg et al. [17].

\section{Immunobloting}

Immunoblot analyses were performed following the method of Kruckeberg et al. [17]. Anti-GFP antibody was purchased from Molecular Probes, and diluted 1:500.

\section{RESULTS}

\section{Construction of a Jen1-GFP fusion protein}

A DNA fragment consisting of the GFP-kanMX6 cassette with short-flanking regions that were homologous with the JEN1 locus at the $3^{\prime}$-end of the ORF was produced by PCR. $S$. 
A

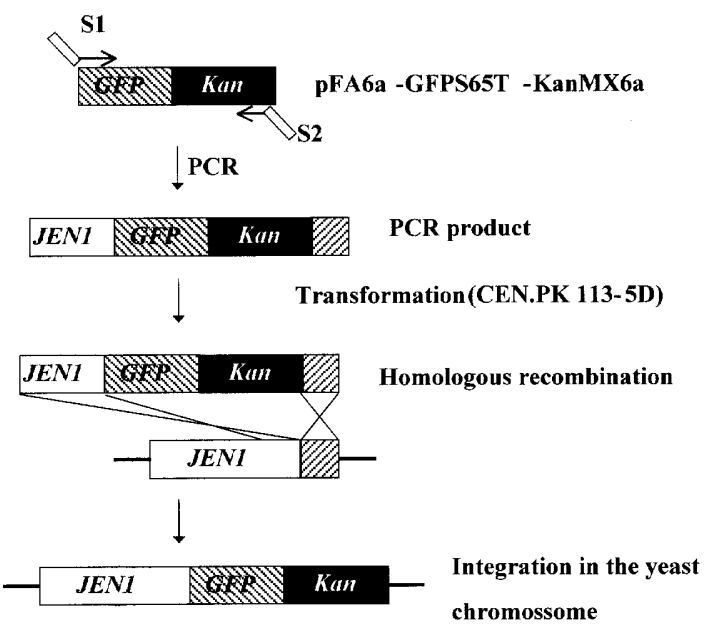

B
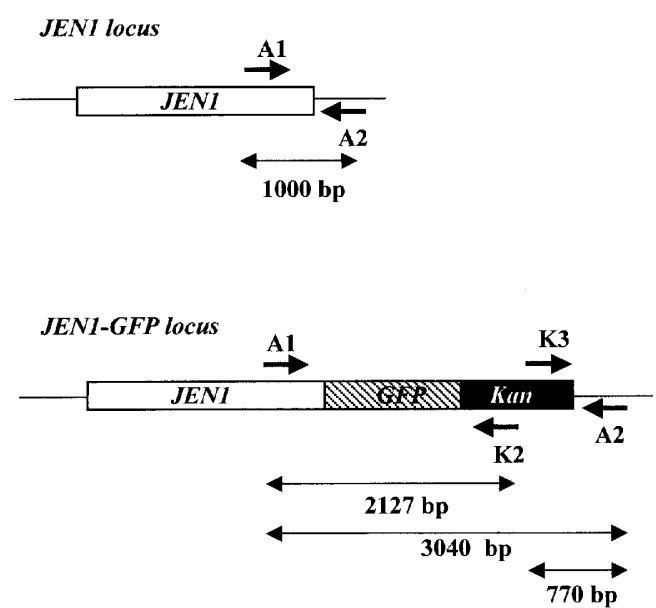

Figure 1 Strategies followed for the construction of the JEN1-GFP fusion (A) and for the verification of gene fusion by analytical PCR (B)

(A) Primers S1 and S2 were used to amplify a GFP-kanMX6 cassette. Primer S1 was homologous with the last 46 nt of JEN1, and primer S2 was homologous with the first 45 nt downstream of JEN1. After transformation of the CEN.PK 113-5D strain with the PCR product, a strain named BLC491-U2 was obtained, bearing the Jen1-GFP fusion protein. (B) Cells from single colonies of BLC491-U2 (JEN1-GFP) and of CEN.PK 113-5D (WT) were lysed as described in the Materials and methods section. Of the cell suspension of each strain, $1 \mu$ l was used directly for PCR analysis according to the previously described strategy. The PCR products were loaded on to a $0.7 \%(\mathrm{w} / \mathrm{v})$ agarose gel, and the size of the fragments obtained are indicated in the Figure.

cerevisiae strain CEN.PK113-5D was transformed with this PCR product to yield the chimaeric $J E N 1-G F P$ gene (Figure 1A). The correct fusion of the targeted gene at the genomic locus was verified by analytical PCR performed on whole cells. The primers used to confirm the correct fusion are summarized in Table 2, and shown in Figure 1(B). Six independent PCRs were performed, with three of them using the strain CEN.PK113-5D as a control and primer pairs $\mathrm{A} 1$ and $\mathrm{A} 2, \mathrm{~A} 1$ and $\mathrm{K} 2$, and $\mathrm{A} 2$ and $\mathrm{K} 3$. Using the same primers, three PCRs were performed using the strain BLC491-U2, bearing the JEN1-GFP fusion. The analytical PCR results clearly confirmed that the PCR-generated DNA fragment was correctly integrated into the CEN.PK113-5D genomic JEN1 locus.

\section{Jen1 fused with GFP is a functional lactate transporter}

De-repressed cells of the strains CEN.PK113-5D and BLC491U2 were obtained under the conditions described in the Materials and methods section, and after $4 \mathrm{~h}$ in YNB/lactic acid medium the cells were analysed for their capacity to transport labelled lactic acid. The results obtained at pH 5.0 are presented in Figure 2 , showing that both strains display activity for the lactate permease. The kinetic parameters observed were of the same order of magnitude: for the CEN.PK113-5D strain, $V_{\max }$ was estimated to be $0.28 \mathrm{nmol} / \mathrm{s}$ per mg of dry mass, and $K_{\mathrm{m}}$ was estimated to be $0.44 \mathrm{mM}$; for the BLC491-U2 strain, $V_{\max }$ was estimated to be $0.30 \mathrm{nmol} / \mathrm{s}$ per $\mathrm{mg}$ of dry mass, and $K_{\mathrm{m}}$ was estimated to be $0.49 \mathrm{mM}$. Under the same experimental conditions, transport of labelled lactic acid was evaluated in cells not expressing JEN1 (strain CEN.PK 113-13D-Ajenl). In this strain, the initial uptake rates were significantly lower than those observed in cells expressing JEN1. Furthermore, first-order kinetics were found, indicative of the absence of a mediated transport mechanism for the acid across the plasma membrane (Figure 2). Cells of the strain W303-1a, obtained under identical experimental conditions [1], displayed similar kinetic parameters:

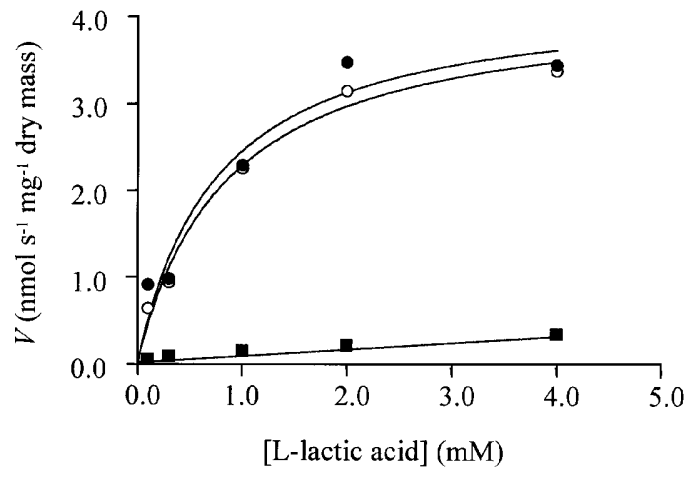

Figure 2 Initial uptake rates of labelled lactic acid at pH 5.0 by YNB/lactic acid-derepressed cells of various yeast strains

Shown are the results of experiments performed with strains CEN.PK 113-5D (O), BLC491U2 $(\bigcirc)$ and CEN.PK $113-13 D-\Delta j e n 1(\square)$. Uptake studies were performed as described in the Materials and methods section. Cells that were growing exponentially in YNB-glucose were harvested by centrifugation, washed twice with deionized water, and incubated in YNB-lactate for $4 \mathrm{~h}$ before the transport assay.

a $V_{\max }$ of $0.40 \mathrm{nmol} / \mathrm{s}$ per $\mathrm{mg}$ of dry mass and a $K_{\mathrm{m}}$ of $0.69 \mathrm{mM}$. Cultures of strains CEN.PK113-5D and BLC491-U2 were grown in YNB-glucose and YNB-lactic acid liquid and solid media. After $48 \mathrm{~h}$, no differences were found between them in terms of the final biomass or the growth rates.

\section{Jen1-GFP is localized in the plasma membrane}

The expression and subcellular localization of Jen1-GFP fusion protein was monitored over time in living cells by fluorescence microscopy. BLC491-U2 cells were grown overnight in YNBglucose medium until they reached a $D_{600}$ of approx. 0.5 , and 
Time (h)

2

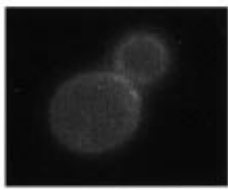

2.5

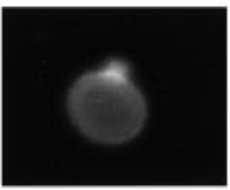

3

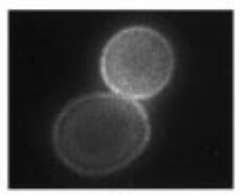

3.5

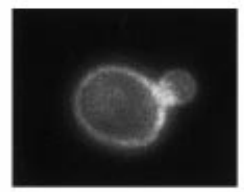

5.5

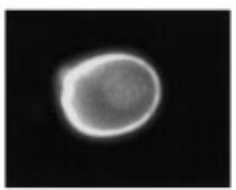

6.5
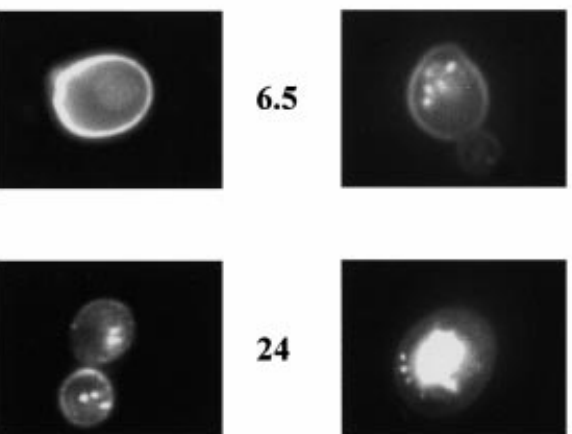

24

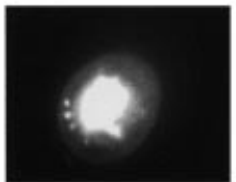

Figure 3 Photographs of a time-course study showing the localization of fluorescent JEN1-GFP in living cells

Cells growing exponentially in YNB-glucose were harvested by centrifugation, washed twice with deionized water, and incubated in YNB-lactate. Equal volumes of cells were resuspended in lowmelt agarose and observed by epifluorescence microscopy. Photos were then taken at the times indicated.

A

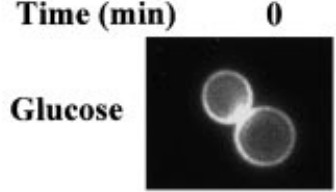

Sorbitol

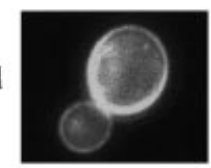

5
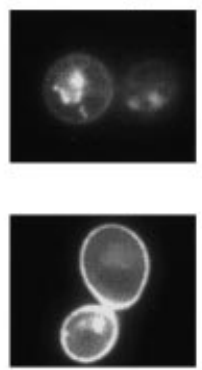

10
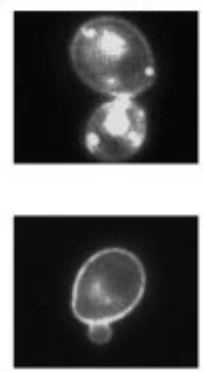

15
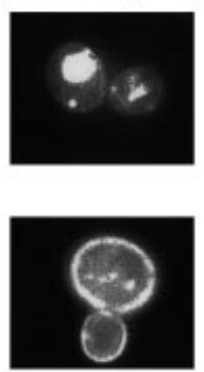

30
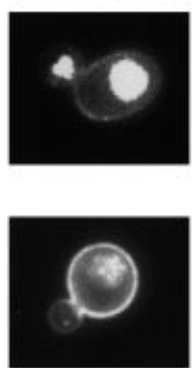

60
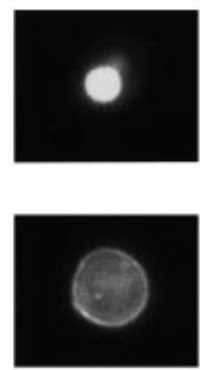

1 h PC

B
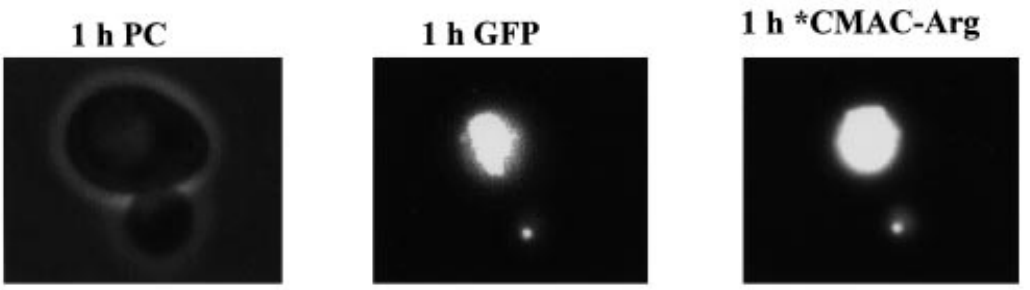

Figure 4 Time course of JEN1-GFP inactivation

(A) Induced cells of the BLC491-U2 strain were treated with glucose or with sorbitol (as a control for osmotic shock), to a final concentration of 110 mM, and examined after continued incubation. (B) Induced cells of the BLC491-U2 strain were examined after 1 h of glucose treatment (final concentration 110 mM) and stained with CMAC-arginine, either detecting GFP fluorescence or CMACarginine fluorescence [17]. CMAC-arginine is a vacuole-specific stain. PC, phase contrast.

were subsequently transferred to YNB medium containing lactic acid $[0.5 \%(\mathrm{w} / \mathrm{v})$ at $\mathrm{pH} 5.0]$ as the sole carbon and energy source. After relocating the cells to medium with lactic acid, the expression and localization of Jen1-GFP, the lactic acid concentration and the attenuance of the culture were measured simultaneously over a $24 \mathrm{~h}$ period. In Figure 3, representative photographs of Jen1-GFP fluorescence in cells at various time points are shown. The fluorescence was almost undetectable within the first $2 \mathrm{~h}$. After that, it started to become clearly localized to the plasma membrane, and increased gradually. The maximum fluorescence intensity in the plasma membrane was achieved 4-6 h after induction of the medium with lactic acid. 
$23{ }^{\circ} \mathrm{C}$

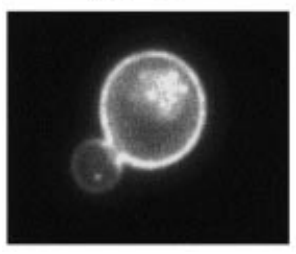

$37^{\circ} \mathrm{C}$

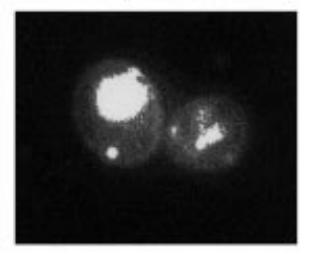

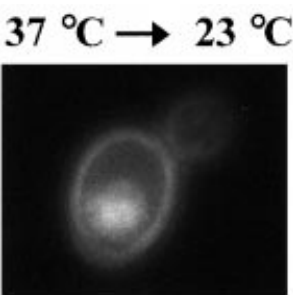

Figure 5 Jen1-GFP localization in a secretory mutant

Jen1-GFP expression was induced in the BLC492 strain. BLC492 strain was derived from the parental cross between BLC491-U2 strain and a sec6-1 strain with a temperature-sensitive defect in fusion of the secretory vesicles with the plasma membrane. Cells were induced in YNB-containing lactic acid $(0.5 \%$, v/v, pH 5.0$)$ for $4 \mathrm{~h}$ at the permissive temperature $\left(23{ }^{\circ} \mathrm{C}\right)$ or at the restrictive temperature $\left(37^{\circ} \mathrm{C}\right)$. After this assay, the cells induced at the restrictive temperature were treated with $10 \mu \mathrm{g} / \mathrm{ml}$ cycloheximide, and incubated at $23^{\circ} \mathrm{C}$ for a further $2 \mathrm{~h}$.

After a prolonged incubation, the signal was lost from the plasma membrane, and the Jen1-GFP fusion protein began to be internalized by endocytosis, and was subsequently delivered to the vacuole. The metabolite content present in the medium culture was examined by HPLC, and it was found that consumption of lactic acid occurred during the whole experiment (results not shown).

\section{Catalytic-centre activity of the Jen1-GFP lactate transporter}

Light absorption and fluorescence emission spectra were recorded from cell suspensions expressing JEN1 or JEN1-GFP. The specific fluorescence of purified GFP is indistinguishable from membrane protein-GFP fusions in vivo (A. Kruckeberg, unpublished results). The fluorescent signal from Jen1-GFP was used to determine the cellular concentration and catalytic-centre activity of the fusion protein, using purified GFP as a fluorescent standard. The cellular abundance of the Jen1-GFP chimaera proved to be in 1670 molecules/cell, and the estimated value for the catalytic-centre activity was $123 \mathrm{~s}^{1}$. All the fluorescence signal appeared to reside at the plasma membrane in the cells expressing JEN1-GFP, when examined by fluorescence microscopy (results not shown).

\section{Inactivation of Jen1-GFP by glucose}

Cells of the strain BLC491-U2 induced with lactate were treated with glucose or sorbitol (final concentration $110 \mathrm{mM}$ ), and examined after a continued period of incubation (Figure 4A). In glucose-treated cells, after 5 min the fluorescent signal was almost completely lost from the plasma membrane, and appeared in punctuate structures. After 30 min of incubation, it accumulated in a single, large globular structure. In sorbitol-treated cells, the fluorescence remained in the plasma membrane. To identify the globular structure observed in cells after $30 \mathrm{~min}$ of glucose addition, the cells were incubated with CMAC-Arg, which stains the vacuole [17]. The GFP fluorescence clearly co-localized with vacuoles stained by CMAC-Arg (Figure 4B).

\section{Jen1-GFP is targeted to the plasma membrane via a Sec6- dependent process}

The role of the secretory pathway in the trafficking of Jen1-GFP to the plasma membrane was assessed in a strain with a temperature-sensitive allele of the SEC6 gene [19,20]. The growth of 10 complete tetrads originating from the parental cross between the BLC491-U2 and $\Delta$ sec6-4 strains was evaluated on YPD medium, both at the permissive temperature of $23{ }^{\circ} \mathrm{C}$ and at the restrictive temperature of $37^{\circ} \mathrm{C}$, and on YPD medium supplemented with Geneticin. One haploid strain, BLC492, was selected, which exhibited normal growth on YPD+geneticin, and which was temperature-sensitive at $37^{\circ} \mathrm{C}$. When the expression of the Jen1-GFP fusion protein was induced in this strain grown at the permissive temperature, the plasma membrane was clearly labelled by GFP (Figure 5). When the fusion protein was expressed in cells maintained at the restrictive temperature of $37^{\circ} \mathrm{C}$, the fluorescence developed to a similar level, but it was completely excluded from the plasma membrane. Instead, it accumulated in globular bodies within the cell (Figure 5). The sec6-4 phenotype has been reported to be reversible, when cells are restored to the permissive temperature [19]. After sec6-4 cells expressing Jen1-GFP were shifted from the restrictive to the permissive temperature for $2 \mathrm{~h}$, their plasma membranes were distinctly labelled with fluorescence (Figure 5). This assay was performed in the presence of cycloheximide in order to prevent the de novo synthesis of protein.

\section{Endocytosis is involved in the removal of Jen1-GFP from the plasma membrane}

Strains with the end3-1 or end4-1 alleles display temperaturesensitive defects in endocytosis [10]. The growth of 10 complete tetrads originating from the parental cross between BLC491-U2 and an end3-1 strain was evaluated on YPD medium, both at the permissive temperature of $23^{\circ} \mathrm{C}$ and at the restrictive temperature of $37^{\circ} \mathrm{C}$ and on YPD supplemented with Geneticin. One haploid strain, BLC493, was selected, which exhibited normal growth on YPD + Geneticin medium, and which was temperature-sensitive at $37^{\circ} \mathrm{C}$. The expression of $J E N 1-G F P$ was induced for $4 \mathrm{~h}$, both in the BLC493 (end3-1) strain and in the BLC491-U2 strain at $23{ }^{\circ} \mathrm{C}$. After $4 \mathrm{~h}$, there was a clear localization of the fluorescence to the plasma membrane in both strains (Figure 6). Each culture was then divided into four aliquots. Two were maintained at the permissive temperature, and two were transferred to the restrictive temperature. Glucose was added to one of the aliquots to a final concentration of $2 \%(\mathrm{w} / \mathrm{v})$ at each temperature. All the cultures were visualized by fluorescence microscopy after $30 \mathrm{~min}$ (Figure 6). In the absence of glucose, there was a strong fluorescent staining of the plasma membrane in all cultures, both at $23{ }^{\circ} \mathrm{C}$ and at $37^{\circ} \mathrm{C}$. The addition of glucose led to an accumulation of the fluorescence in the vacuole both in BLC491$\mathrm{U} 2$ and in BLC493 cells at the permissive temperature, but only in BLC491-U2 cells at the restrictive temperature. In BLC493 cells at $37^{\circ} \mathrm{C}$, there was a retention of JEN1-GFP in the plasma membrane, which persisted with prolonged incubation. Taken together, these results indicate that, upon glucose treatment, 


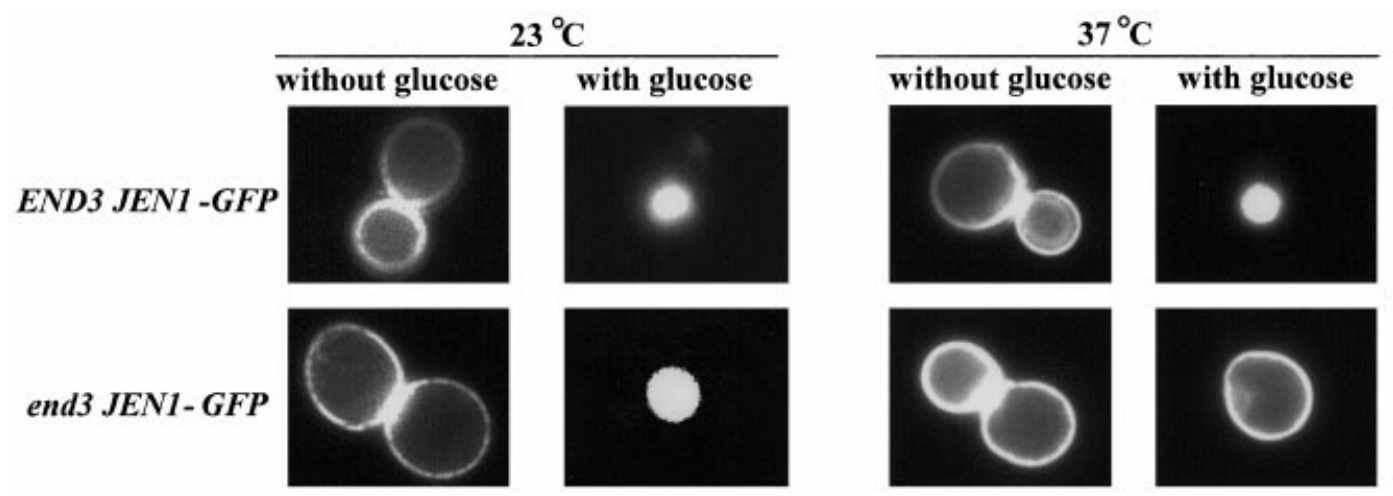

Figure 6 Jen1-GFP is retained in the plasma membrane in cells blocked in endocytosis

Jen1-GFP expression was induced for $4 \mathrm{~h}$, in both the BLC493 and BLC491-U2 strains at $23^{\circ} \mathrm{C}$, under the conditions described in the Materials and methods section. After $4 \mathrm{~h}$ of induction, each culture was divided into four aliquots. Two were maintained at the permissive temperature, and two were transferred to the restrictive temperature. Glucose was added to one of the aliquots at each temperature to a final concentration of $2 \%(\mathrm{w} / \mathrm{v})$. All the cultures were visualized by fluorescence microscopy after $1 \mathrm{~h}$.

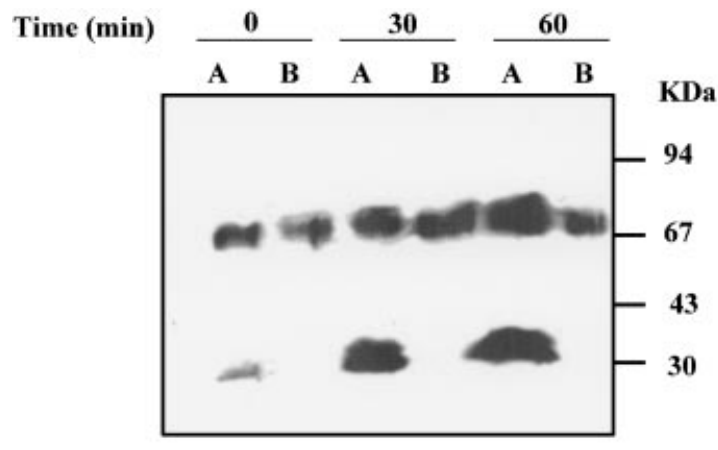

Figure 7 Western-blot analysis of Jen1-GFP protein level

The strains used were BLC491-U2 (END3 JEN1-GFP) (lanes labelled 'A') and BLC 493 (end3 JEN1-GFP) (lanes labelled 'B'). Detection of Jen1-GFP was performed with anti-GFP antibody. Time after glucose addition is shown.
Jen1-GFP is endocytosed via END3, and subsequently targeted to the vacuole.

Western blot analysis of the effect of glucose addition in cells blocked in endocytosis

A Western blot analysis was performed using an anti-GFP antibody. The strains BLC 491-U2 and BLC-493 were induced in lactate for $4 \mathrm{~h}$. After this period of induction, a sample of each culture was collected and glucose was added to the culture medium to a final concentration of $2 \%(\mathrm{w} / \mathrm{v})$. Of each culture, two more samples were collected after 30 and $60 \mathrm{~min}$ of glucose addition. Lysates containing $15 \mu \mathrm{g}$ of protein were resolved on an SDS $/ 10 \%$ polyacrylamide gel. After blotting, the degradation products were only observed in the BLC491-U2 strain (Figure 7). No degradation products could be observed in the strain with a defect in the endocytic pathway, supporting the results previously obtained by fluorescence microscopy (Figure 7).

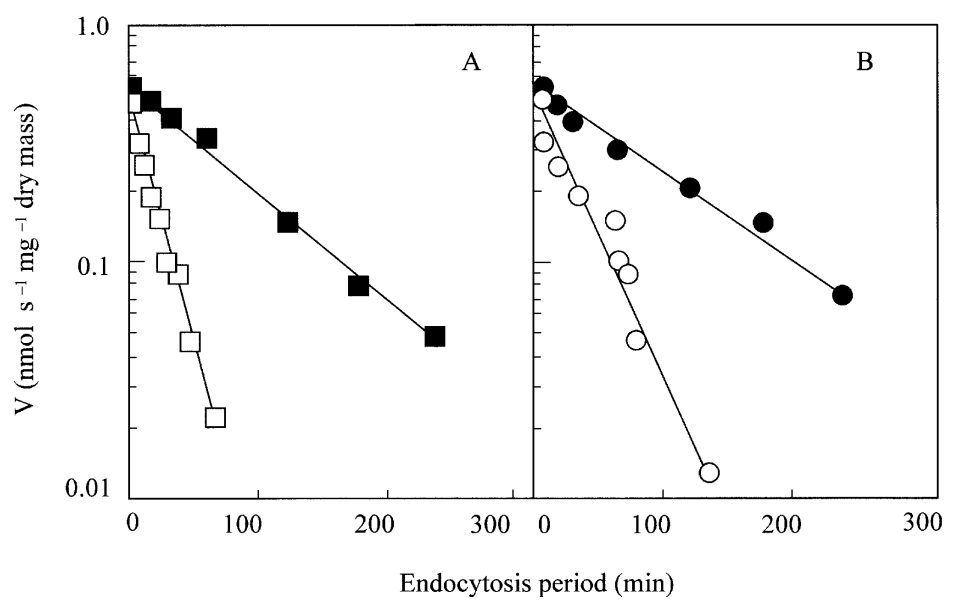

Figure 8 Activity of the lactate transporter in induced cells upon the addition of glucose, using $1 \mathrm{mM}$ of labelled lactic acid, pH 5.0

(A) Activities are shown for wild-type cells $(\square)$ and $\Delta$ doa4 cells ( (B) $\Delta$ doa4 cells transformed with the plasmid YEp96, carrying the wild-type Ub gene, were induced in the presence $(O)$ or absence $\left(\mathbf{)}\right.$ ) of $\mathrm{Cu}^{2+}$. The final glucose concentration was $2 \%(\mathrm{w} / \mathrm{v})$. 


\section{Overexpression of Ub partially restored endocytosis of the lactate transporter in a $\Delta d 0 a 4$ strain}

We then set out to determine whether the Ub pathway was involved in the endocytosis of the lactate permease upon glucose treatment. The Doa4p Ub-isopeptidase has been shown to have a key role in Ub-dependent degradation in vivo [21], and a doa4 mutant has reduced levels of free $\mathrm{Ub}$ [21]. We used the yeast strain MHY623 lacking the Ub protein hydrolase Doa4/Npi2. Cultures of induced cells of MHY501 and MHY623 strains were supplemented with glucose to a final concentration of $2 \%(\mathrm{w} / \mathrm{v})$, and samples were collected over time to estimate the activity of the carrier. Upon glucose treatment, the inactivation of the carrier is substantially reduced in a $\Delta$ doa4 strain when compared with the wild type (Figure $8 \mathrm{~A}$ ). These results suggest that the internalization step of endocytosis of the Jen 1 permease is dependent on the Doa4 protein. The $\Delta$ doa4 phenotype can be complemented with an overproduction of Ub [22]. This can be achieved by transforming the mutant strain with the plasmid YEp96. This multi-copy plasmid encodes a synthetic $U b$ gene under the control of the CUPI-inducible promoter. The overexpression of Ub 'rescued' the internalization of the permease in $\Delta$ doa 4 cells. However, in the presence of copper, no effect was found, in comparison with that which was observed for the strain $\Delta$ doa4 not transformed with YEp96 (Figure 8B).

\section{DISCUSSION}

The properties and regulation of solute transporter proteins in vivo have been investigated further by tagging them with the GFP of $A$. victoria. The analysis of a number of $S$. cerevisiae solute transporter proteins as fusion proteins with GFP has been reported previously: some of the tagged proteins are members of sugar-transporter family [3,23]. Furthermore, the hexose transporters Hxt2 [17] and Hxt7 [24], and the Pho84 phosphate transporter [25] have been tagged with GFP. In all cases tested, the fusion proteins retain solute transport function, with kinetics similar to those of the wild-type protein. The genetic chimaera formed between Jen 1 and GFP is also a functional lactate transporter. The expression pattern and intracellular trafficking of the fusion protein, monitored by fluorimetry and epifluorescence microscopy, has been determined. We observed that the protein is strongly localized at the plasma membrane in induced cells. The maximum expression at the plasma membrane was obtained between 4-6 h after induction, and this result was in accordance with the results obtained previously by determining the activity of the carrier [26].

The quantification of the emission from GFP in the Jen1-GFP protein allowed us to estimate a value of $123 \mathrm{~s}^{1}$ for the catalyticcentre activity of the lactate transporter in vivo. This is the first empirical estimate of a catalytic-centre activity for a yeast monocarboxylate transport protein. The value obtained is of the same order of magnitude as the one obtained for the GLUT1 human glucose transporter at $37{ }^{\circ} \mathrm{C}$ [27], and for the Hxt7-GFP transporter [24]. All of these solute permeases are high-affinity transporters. The value obtained seems to reflect a high activity of this transporter molecule in cells under induction conditions.

The factors involved in proper localization and turnover of the Jen 1 protein were also explored by expression of the Jen1-GFP fusion in a set of strains with mutations affecting specific steps in the secretory and endocytic pathways. We have shown that SEC6 is involved in delivering Jen1-GFP to the membrane.

The results obtained in a strain defective in the END3 gene indicate that endocytosis is the mechanism involved in the process of catabolite inactivation of the carrier. Glucose triggers a rapid degradation of the lactate permease in the vacuole. Upon treatment with glucose, Jen $1 p$ was removed from the membrane, internalized by endocytosis and accumulated in the vacuole for degradation. The data resulting from the inactivation of Jen1GFP by glucose, together with the results observed in the timecourse experiments (Figure 3), indicate that the permease constitutively undergoes a moderate rate of turnover, in addition to a rapid, stress-stimulated turnover.

We used a strain defective in the Ub-protein hydrolase Doa4/Npi to determine whether the binding of Ub was a signal required for the internalization of the carrier. Jen $1 p$ undergoes internalization for vacuolar degradation in a manner dependent on Doa4p. This pattern of behaviour is also reported in the literature for other plasma membrane proteins whose internalization is substantially reduced in doa4 mutant cells [28-32].

The results obtained with cells featuring impairments in the Doa4 protein showed that ubiquitination of the lactate permease signals its endocytosis. These observations taken together reinforce previous evidence that indicated that there is a general pattern in the mechanism of endocytosis followed by all the plasma membrane proteins studied to date in yeast, as has been suggested by Hicke [33]. The results obtained in the present study suggest that glucose-induced proteolytic degradation (catabolite inactivation) of Jen $1 \mathrm{p}$ seems to occur independently of the proteasome, i.e. it occurs in the vacuole after internalization by glucose. Future experiments will be designed to determine the type of ubiquitination that the Jen1p permease undergoes in $S$. cerevisiae, and the mechanisms that regulate the endocytosis of this transporter.

We thank Professor Karel van Dam and his laboratory for their generous assistance. We also thank Professor Cecília Leão for a critical reading of the manuscript and for valuable discussions. We are grateful to Professor Rosario Lagunas and Dr Pilar Lucero for their helpful information and suggestions. Sandra Paiva received a Ph.D. fellowship from the Portuguese Government (PRAXIS XXI-BD/1898/98) and a FEBS short-term fellowship for the work performed in The Netherlands. This work was supported by the Portuguese grant POCTI/1999/BME/36 625.

\section{REFERENCES}

1 Casal, M., Paiva, S., Andrade, R. P., Gancedo, C. and Leão, C. (1999) The lactateproton symport of Saccharomyces cerevisiae is encoded by JEN1. J. Bacteriol. 181, 2620-2623

2 Makuc, J., Paiva, S., Schauen, M., Kramer, R., André, B., Casal, M., Leão, C. and Boles, E. (2001) The putative monocarboxylate permeases of the yeast Saccharomyces cerevisiae do not transport monocarboxylic acids across the plasma membrane. Yeast 18, 1131-1143

3 Nelissen, B., De Wachter, R. and Goffeau, A. (1997) Classification of all putative permeases and other membrane plurispanners of the major facilitator superfamily encoded by the complete genome of Saccharomyces cerevisiae. FEMS Microbiol. Rev. 21, 113-134

4 Paulsen, I. T., Sliwinski, M. K., Nelissen, B., Goffeau, A. and Saier, Jr, M. H. (1998) Unified inventory of established and putative transporters encoded within the complete genome of Saccharomyces cerevisiae. FEBS Lett. 23, 116-125

5 Halestrap, A. P. and Price, N. T. (1999) The proton-linked monocarboxylate transporter (MCT) family: structure, function and regulation. Biochem. J. 343 281-299

6 Andrade, R. P. and Casal, M. (2001) Expression of the lactate permease gene JEN1 from the yeast Saccharomyces cerevisiae. Fungal Genet. Biol. 32, 105-111

7 Tsien, R. Y. (1998) The green fluorescent protein. Annu. Rev. Biochem. 67, 509-544

8 Giuseppin, M. L., Heijnen, J. J., Hoare, M., Lange, H. C., Madden, E. A., Niederberger, P., Nielsen, J., Parrou, J. L., Petit, T., Porro, D. et al. (2000) An interlaboratory comparison of physiological and genetic properties of four Saccharomyces cerevisiae strains. Enzyme Microb. Technol. 26, 706-714

9 Nakamoto, R. K., Rao, R. and Slayman, W. (1991) Expression of the yeast plasma membrane $\mathrm{H}^{+}$-ATPase in secretory vesicles. A new strategy for direct mutagenesis. J. Biol. Chem. 266, 7940-7949

10 Raths, S., Rohrer, J., Crausaz, F. and Riezman, H. (1993) end3 and end4: two mutants defective in receptor-mediated and fluid-phase endocytosis in Saccharomyces cerevisiae. J. Cell Biol. 120, 55-65 
11 Hochstrasser, M., Ellison, M. J., Chau, V. and Varshavsky, A. (1995) The short-lived Mata2 transcriptional regulator is ubiquitinated in vivo. Proc. Natl. Acad. Sci. U.S.A. 88, $4406-4610$

12 Reference deleted

13 Sherman, F. and Hicks, J. (1991) Micromanipulation and dissection of asci. Methods Enzymol. 194, 21-37

14 Wach, A., Brachat, A., Pohlmann, R. and Philippsen, P. (1994) New heterologous modules for classical or PCR-based gene disruptions in Saccharomyces cerevisiae. Yeast 10, 1793-1808

15 Wach, A., Brachat, A., Alberti-Segui, C., Rebischung, C. and Philippsen, P. (1997) Heterologous HIS3 marker and GFP reporter modules for PCR-targeting in Saccharomyces cerevisiae. Yeast 13, 1065-1075

16 Gietz, R. D., Schiestl, R. H., Willems, A. R. and Woods, R. A. (1995) Studies on the transformation of intact yeast cells by the LiAc/SS-DNA/PEG procedure. Yeast 11, $355-360$

17 Kruckeberg, A. L., Ye, L., Berden, J. A. and van Dam, K. (1999) Functional expression, quantification and cellular localization of the Hxt2 hexose transporter of Saccharomyces cerevisiae tagged with the green fluorescent protein. Biochem. J. $\mathbf{1 5}$ 299-307

18 Sambrook, J., Fritsch, E. F. and Maniatis, T. (1989) Molecular cloning: a laboratory manual. 2nd edn, Cold Spring Harbor Laboratory Press, New York

19 Novick, P., Field, C. and Schekman, R. (1980) Identification of 23 complementation groups required for post-translational events in the yeast secretory pathway. Cell (Cambridge, Mass.) 21, 205-215

20 Tschopp, J., Esmon, P. C. and Schekman, R. (1984) Defective plasma membrane assembly in yeast secretory mutants. J. Bacteriol. 160, 966-970

21 Papa, F. R. and Hochstrasser, M. (1993) The yeast DOA4 gene encodes a deubiquitinating enzyme related to a product of the human tre-2 oncogene. Nature (London) 366, 313-319

Received 28 November 2001/30 January 2002; accepted 15 February 2002
22 Baker, R. T., Gilchrist, C., Wyndham, A., Wang, X. W. and Johnson, E. (1995) Role of ubiquitin specific proteases in protein degradation. Yeast 11, 349

23 Kruckeberg, A. L. (1996) The hexose transporter family of Saccharomyces cerevisiae. Arch. Microbiol. 166, 283-292

24 Ye, L., Berden, J. A., van Dam, K. and Kruckeberg, A. L. (2001) Expression and activity of the $H x t 7$ high-affinity hexose transporter of Saccharomyces cerevisiae. Yeast 18, 1257-1267

25 Petersson, J., Pattison, J., Kruckeberg, A. L., Berden, J. A. and Persson, B. L. (1999) Intracellular localization of an active green fluorescent protein-tagged Pho84 phosphate permease in Saccharomyces cerevisiae. FEBS Lett. 462, 37-42

26 Casal, M., Cardoso, H. and Leão, C. (1996) Mechanisms regulating the transport of acetic acid in Saccharomyces cerevisiae. Microbiology 142, 1385-1390

27 Maher, F., Davies-Hill, T. M. and Simpson, I. A. (1996) Substrate specificity and kinetic parameters of GLUT3 in rat cerebellar granule neurons. Biochem. J. 315, $827-831$

28 Galan, J. M. and Haguenauer-Tsapis, R. (1997) Ubiquitin lys63 is involved in ubiquitination of a yeast plasma membrane protein. EMBO J. 16, 5847-5854

29 Lucero, P. and Lagunas, R. (1997) Catabolite inactivation of the yeast maltose transporter requires ubiquitin-ligase npi1/rsp5 and ubiquitin-hydrolase npi2/doa4. FEMS Microbiol. Lett. 15, 273-277

30 Medintz, I., Jiang, H. and Michels, C. A. (1998) The role of ubiquitin conjugation in glucose-induced proteolysis of Saccharomyces maltose permease. J. Biol. Chem. 273, $34454-34462$

31 Springael, J. Y., Galan, J. M., Haguenauer-Tsapis, R. and Andre, B. (1999) $\mathrm{NH}^{+}$. induced down-regulation of the Saccharomyces cerevisiae Gap1p permease involves its ubiquitination with lysine-63-linked chains. J. Cell Sci. 112, 1375-1383

32 Terrell, J., Shih, S., Dunn, R. and Hicke, L. (1998) A function for monoubiquitination in the internalization of a $\mathrm{G}$ protein-coupled receptor. Mol. Cell 1, 193-202

33 Hicke, L. (1999) Gettin' down with ubiquitin: turning off cell-surface receptors, transporters and channels. Trends Cell Biol. 9. 107-112 